\title{
DYNAMIC CHANGE MONITORING OF QIANLONG GARDEN ROCKERY IN FORBIDDEN CITY BASED ON INSAR AND LIDAR TECHNOLOGY
}

\author{
Guo Ming ${ }^{1,2,3,4}$, Zhou Yuquan ${ }^{1}$, Zhou Tengfei ${ }^{1}$, Pan Deng ${ }^{5, *}$ \\ ${ }^{1}$ Institute of Mapping and Urban Spatial Information, Beijing University of Civil Engineering and Architecture, Beijing 100044, \\ China; \\ ${ }^{2}$ Engineering Research Center of Representative Building and Ancient Architecture Database, The Ministry of Education, Beijing \\ 102616, China; \\ ${ }^{3}$ Beijing Key Laboratory for Architectural Heritage Fine Reconstruction \& Health Monitoring, Beijing 102616, China; \\ ${ }^{4}$ The Key Laboratory of the National Geographic Information Bureau of National Surveying and Mapping in Modern Urban \\ Surveying and Mapping ,102616, China \\ ${ }^{5}$ Beijing University of Civil Engineering and Architecture, School of Civil and Transportation Engineering, Beijing,102616, China;
}

KEY WORDS: Qianlong Garden Rockery, InSAR, PS-InSAR, LiDAR, Deformation Monitoring

\begin{abstract}
:
The traditional method of deformation monitoring involves a large amount of external field measurement and internal processing to obtain the deformation of the measured object. With the development of InSAR technology, deformation monitoring can now be completed more efficiently. In this paper, InSAR technology and LiDAR technology are used to detect the deformation of the Fuwangge Rockery in Qianlong Garden, InSAR technology can provide higher point accuracy and LiDAR technology can provide higher spatial resolution. After the deformation of the rockery is obtained by LiDAR technology, the InSAR experimental results are verified. It is expected that the error of deformation analysis by the two methods will be within $5 \mathrm{~mm}$. Finally, the detection results of the two are consistent with each other and complement each other. At the same time, InSAR technology combined with LiDAR technology also provides a new idea for deformation monitoring.
\end{abstract}

\section{INTRODUCTION}

Deformation monitoring is the use of special instruments and methods to continuously observe the deformation phenomenon of the deformation body. It can analyze the deformation shape of the deformation body and predict the change trend of the deformation body. Deformation monitoring includes the establishment of a deformation detection network for horizontal displacement, settlement, tilting, cracking, deflection, oscillation and vibration monitoring. Compared with conventional measurement, a remarkable feature of deformation monitoring is the high measurement accuracy. The general monitoring accuracy should also reach millimetre level. The important and sensitive deformation should reach $0.1 \mathrm{~mm}$ or even $0.01 \mathrm{~mm}$. Therefore, deformation monitoring is mostly a precision measurement. Deformation monitoring is of great significance, rich in content, high precision, and has many differences with topographic surveys, construction surveys et al. and has a relatively independent technical system, which has developed into a highly specialized sub-discipline in surveying (Guo, 2013). As the price of 3D laser scanners declines, 3D laser scanning technology is becoming more and more mature. The ground 3D laser scanning technology has been widely used in urban planning, cultural relics protection, restoration of ancient buildings, transportation and deformation monitoring (Zhu, 2013; Zhang and Cheng, 2018; Chang et al., 2019; Wang et al., 2013; Li et al., 2015; Guo et al., 2020; Wang et al., 2018; Gao et al., 2019). In recent years, the cultural relics protection personnel of the Forbidden City in Beijing used the 3D laser scanner to count the main ancient buildings of the Forbidden City.

\footnotetext{
* Corresponding author: 18342806590@163.com
}

The word modeling attempts to restore the architecture of the Forbidden City to the original appearance of the Kanggansheng, which provides first-hand data for the restoration of ancient buildings. In 2005, the technicians of the Xi'an 4D Aerial Survey and Remote Sensing Centre completed the scanning of the Terracotta Warriors and Horses No. 2 pit of the Terracotta Warriors and Horses Museum through the use of the Leica HDS3000 and HDS2500 two kinds of dimensional laser scanners, by the use of Cyclone data processing software, completed the scan data splicing and 3D modeling of the entire pit (Yang, 2006). In 2006, Vassilios Pagounis et al. used 3D laser scanners to obtain spatial position information data of various roads and roadsides at road intersections, and used the acquired data for road safety analysis and traffic accident simulation (Pagounis et al., 2006). Deformation monitoring is to measure the monitoring points on the same deformation area in different time periods, and analyze the obtained data to determine the shape, size and position of the deformation body. The space state and time characteristics are mainly used to monitor whether the deformation body is in a safe state (Zhu, 2013)

The study area in this paper was selected as Qianlong Garden. Qianlong Garden (also known as Ningshou Palace Garden) is located in the northwest corner of the Ningshou Palace in the East Road of the Forbidden City. It is part of the Ningshou Palace Complex. Built in the thirty-six to eleventh year of Emperor Qianlong (AD 1771-1776), the garden is 160 meters long from north to south and 37 meters wide from east to west. It faces the palace wall in the west and the palace in the east, covering an area of 5,920 square meters. The layout of the entire garden is exquisite and well-constructed. It is a model of 
the Palace Garden. There are more than 20 buildings, such as pavilions, terraces, pavilions and buildings in the park. The types are rich, the size is different, the conditions are different, the distribution is intricate, and the verandas and the winding corridors make the buildings and flowers and trees cross. The mood is harmonious. Asymmetric processing is adopted on the plane and the façade, and it is particularly dexterous and novel

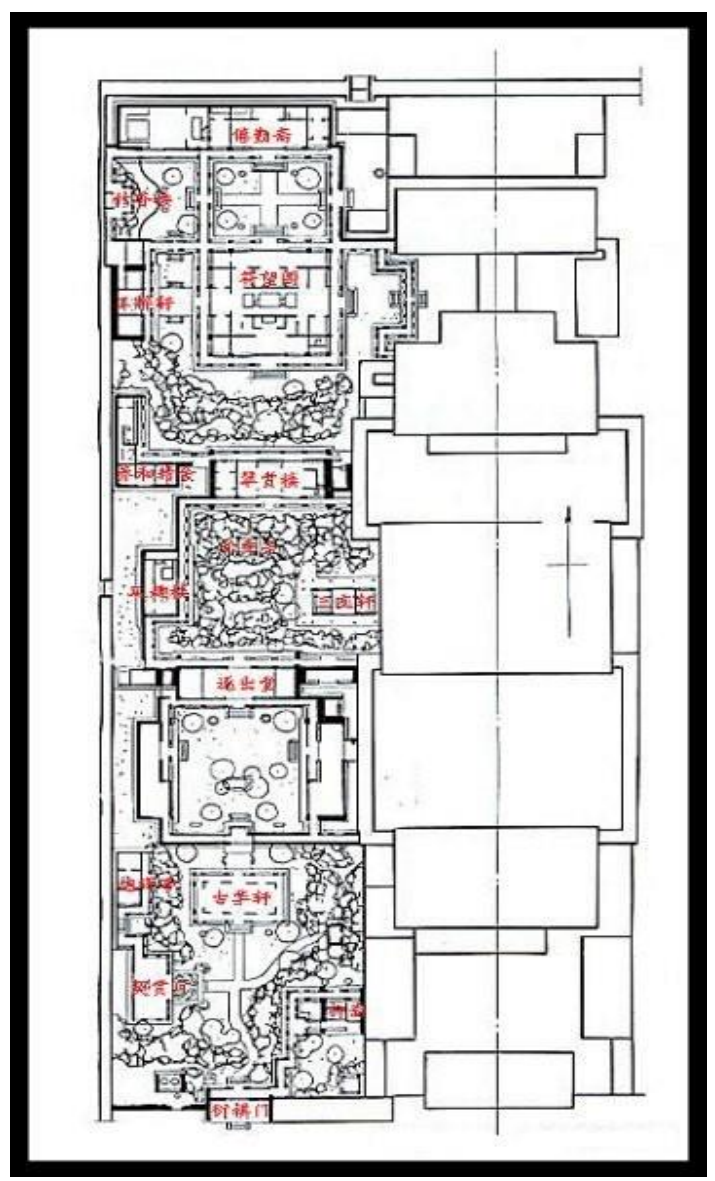

Figure 1. Floor plan of the Forbidden City Qianlong Garden

\section{MATERIALS AND METHODS}

\subsection{COSMO-SkyMed data}

The data used herein is COSMO-SkyMed data. COSMOSkyMed (Constellation of Small Satellites for Mediterranean Basin Observation) is a high-resolution radar satellite constellation jointly developed by the Italian Space Agency and the Ministry of Defence. It consists of four X-band synthetic aperture radar satellites. COSMO-SkyMed (CSK) image data has the characteristics of high geometric resolution, wide coverage, optional angle of incidence, multi-polarization et al., plus precise orbital data, which is very useful in monitoring the deformation of ground objects by using repeated orbital interferometry.

This article uses the CSK image data of the up-track, the track is 99999. The image data is obtained by SAR1 and SAR3, and the polarization mode is $\mathrm{HH}$. The incident angle of the image center is about 26 degrees, and the imaging mode is the strip mode. Among them, the strip mode is divided into two modes: HIMAGE and PingPong. In the HIMAGE mode, the radar transmit antenna and the receive antenna configuration do not in the rigorous ban of the system. The exquisite chic of the garden in the garden and the luxurious and magnificent of the royal palace are the most famous gardens in the four gardens of the Forbidden City (the other three are the Royal Garden, the Cining Palace Garden and the Jianfu Palace Garden). Figure 1 shows the plan of the Forbidden City Qianlong Garden and the Figure 2 shows the landscape of Qianlong Garden.

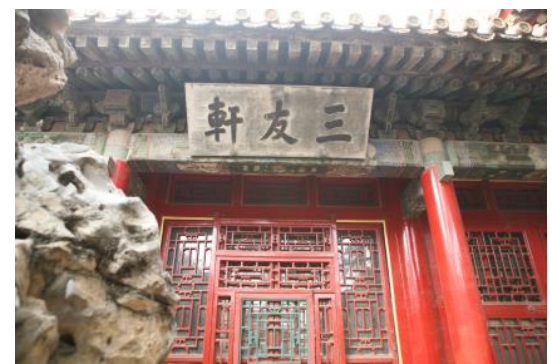

(a)

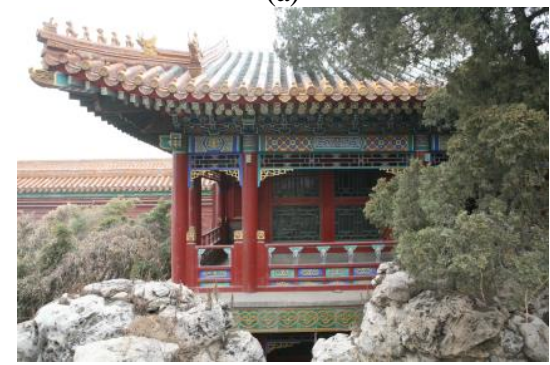

(b)

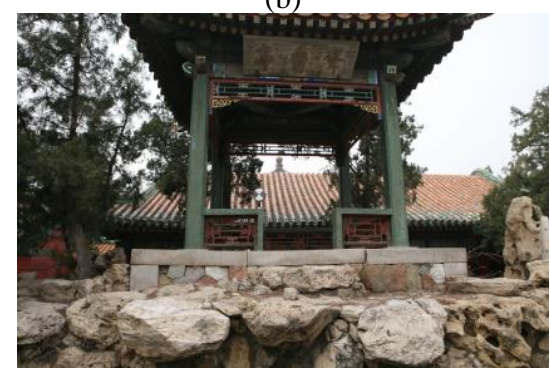

(c)

Figure 2. Landscape of Qianlong Garden (a)Sanyouxuan; (b)Yanqulou; (c) Songxiuting

change with time, and can receive the full Doppler beam width of each ground scatterer. The HIMAGE mode has a width of about $40 \mathrm{~km}$ and an image resolution of $3 \mathrm{~m}$. Because the highresolution X-band SAR image has shorter wavelengths than the C-band and L-band, it is sensitive to the small shape of the ground object, and it has obvious advantages in detecting the small deformation of the building surface. Table 1 shows the CSK image parameters.

\subsection{InSAR technology}

2.2.1 InSAR data: InSAR (Interferometric Synthetic Aperture Radar), is a fast-developing geodetic technology, which can obtain high-precision and continuous coverage of ground elevation and surface change information all the time. Using two SAR images in the same region as the basic processing data, obtains the interference image by obtaining the phase difference between the two SAR images, and then undergoes phase unwrapping. The terrain elevation data is obtained from the interference fringes, and the minute displacement of the target point is measured according to the phase change information. 


\begin{tabular}{ccccccc}
\hline Numbering & Satellite & $\begin{array}{c}\text { Track } \\
\text { number }\end{array}$ & $\begin{array}{c}\text { Polarization } \\
\text { mode }\end{array}$ & Date & $\begin{array}{c}\text { Vertical } \\
\text { Baseline/m }\end{array}$ & $\begin{array}{c}\text { Time } \\
\text { Baseline/m }\end{array}$ \\
\hline 0 & SAR3 & 14810 & HH & 20110721 & 268.27 & 359 \\
1 & SAR3 & 15284 & HH & 20110822 & -520.35 & 327 \\
2 & SAR3 & 15758 & HH & 20110923 & 88.04 & 295 \\
3 & SAR3 & 16232 & HH & 2011025 & -79.19 & 263 \\
4 & SAR3 & 16943 & HH & 20110212 & 207.13 & 215 \\
5 & SAR1 & 25001 & HH & 20120120 & -372.15 & 176 \\
6 & SAR1 & 25475 & HH & 20120221 & 305.20 & 144 \\
7 & SAR1 & 25949 & HH & 20120324 & -130.77 & 112 \\
8 & SAR1 & 26423 & HH & 20120425 & -915.56 & 80 \\
9 & SAR1 & 26897 & HH & 20120527 & -39.29 & 48 \\
10 & SAR1 & 27371 & HH & 20120628 & -778.00 & 16 \\
11 & SAR1 & 27608 & HH & 20120714 & 0 & 0 \\
\hline
\end{tabular}

Table 1. CSK parameters

2.2.2 InSAR data processing flow: Due to the special imaging principle of SAR images, it is determined that the SAR image data processing process is fundamentally different from the traditional optical remote sensing image processing. After years of exploration and research, In-SAR data has formed a basic process (Qin Liming,2015). As shown in Figure 3.

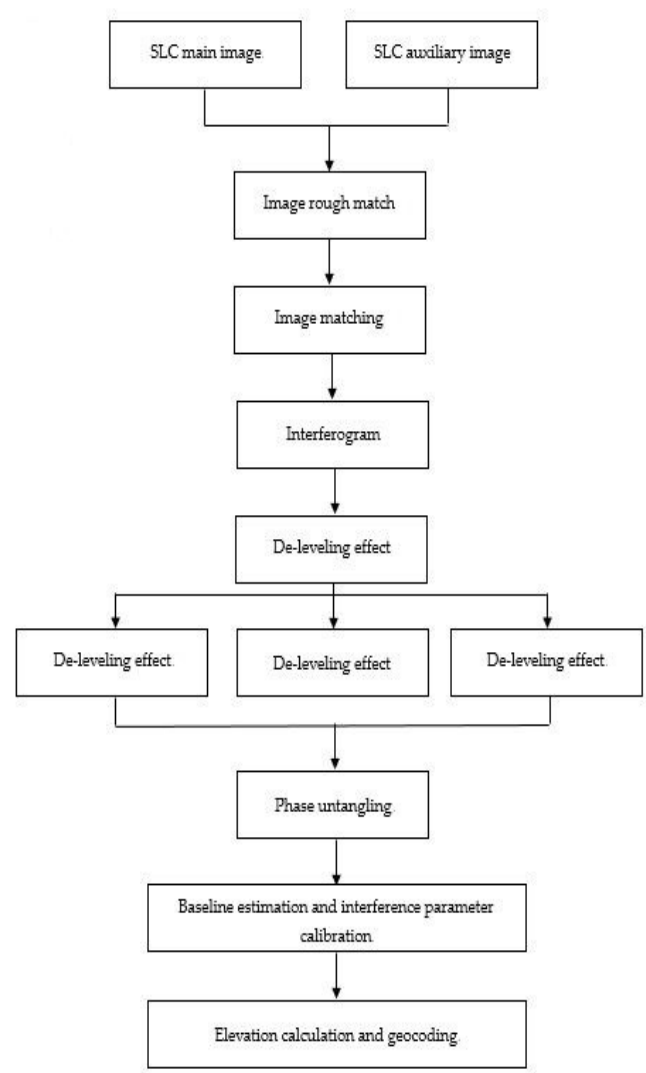

Figure 3. InSAR data processing flow
2.2.3 Deformation information extraction method in InSAR data: There are many methods for deformation monitoring using InSAR data. The most common ones are differential interferometry (D-InSAR), permanent scatterer synthetic aperture radar interferometry (PS-InSAR), small baseline set interferometry (SBAS-InSAR), and distribution. Method of target interference measurement (DS-InSAR) (Zhu et al., 2017; Zhang, 2019; Huang, 2019). The method adopted in this paper is the Permanent Scattering Synthetic Aperture Radar Interferometry (PS-InSAR) method. The literature (Ferrtti et al., 2000; Ferrtti et al., 2001) first proposed the PS-InSAR method. The basic idea is: First, using a multi-view single-view SAR image covering the same study area, one of the scene SAR images is selected as the main image, and the remaining SAR images are respectively. The main image is registered, and the permanent scatterer (PS) target is selected according to the amplitude and/or the stability of the phase information in the time series; secondly, the Interference based on the permanent scatter object is obtained through interference and detopography processing. Interfering with phase and redifferentiating the differential interference phase of adjacent permanent scatterer targets; third, constructing a deformation phase model and spatiotemporal filtering or mode according to different characteristics of each phase component in the interference phase after two differences Estimate deformation and topographic residual information. PS-InSAR technology does not process data for all pixels in SAR images, but selects PS points with relatively stable scattering characteristics and strong echo signals in time as observation objects. These PS points usually include artificial buildings, lighthouses, bare rocks, and manually installed corner reflectors. The accurate selection of the PS points ensures that the PS point exhibits good coherence and stability even under conditions of long interference or long baselines (even reaching a critical baseline). Commonly used PS point selection methods include amplitude dispersion threshold method, coherence coefficient method (Chen, 2006), phase analysis method (Xiong et al., 2015; Jiang, 2018; Diao, 2018), and combinations of these methods. The deformation and terrain residuals are usually solved by the solution space search method, the LAMBDA method (Kampes et al., 2004) and the three-dimensional unwrapping method in the Stam PS (Hopper and Zebker, 2007).At present, PS-InSAR 
technology has been widely used in high-resolution land subsidence monitoring in many cities, especially high-resolution deformation monitoring of urban key infrastructure. By comparing the horizontal level and GPS measurement data, it is proved that PS-InSAR technology has high reliability (mmlevel accuracy) (Li et al., 2004), so it is of great significance to master the dynamic law of urban land subsidence and analyze the cause of ground subsidence. However, PS-InSAR technology also has its own drawbacks. First, it usually requires a large number of SAR images covering the same area (usually required to be larger than 25 scenes), which is convenient for ensuring the reliability of the model solution. Secondly, PSInSAR technology is based on iterative regression or network adjustment calculation of a large number of PS points, and the computational efficiency is not high, so it is not suitable for high-resolution ground subsidence monitoring in a wide range of areas. Finally, the results obtained by PS-InSAR technology include the three-dimensional information and deformation information of the PS point, that is, the observation information of each PS point includes the three-dimensional position information of the PS point, the average deformation rate of the PS point, and the time evolution history of the PS point.

\subsection{LiDAR technology}

2.3.1 LiDAR data: The point cloud data used in this paper are two periods of data in 2010 and 2018 respectively. The 2010 data were collected using Leica ScanStation2 three-dimensional laser scanner and 2018 data were collected using FARO focus 3D130 three-dimensional laser scanner. Some point cloud are shown in Figure 4.

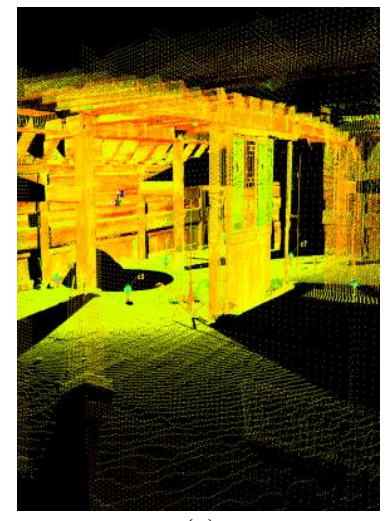

(a)

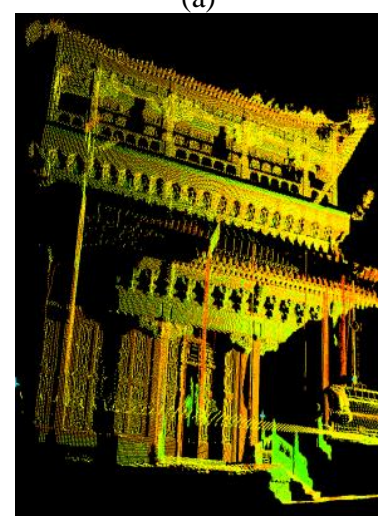

(c)

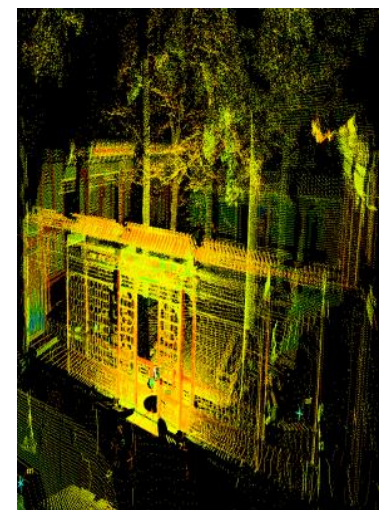

(b)

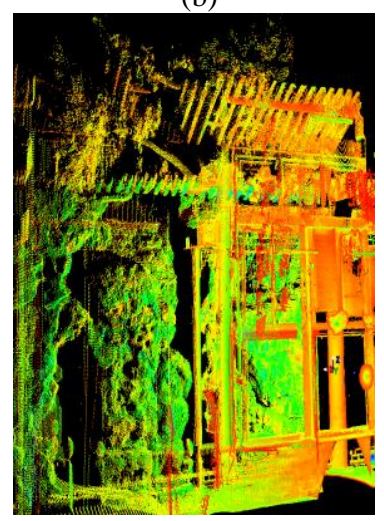

(d)
Figure 4. Point cloud of Qianlong Garden (a) Fuwangge; (b) Juanqinzai; (c) Yuzixuan; (d) Songxiuting
2.3.2 LiDAR data processing flow: The key technologies of point cloud data processing include: multi-view cloud data registration, point cloud data compression, and generation grid data. The specific process is shown in Figure 5.

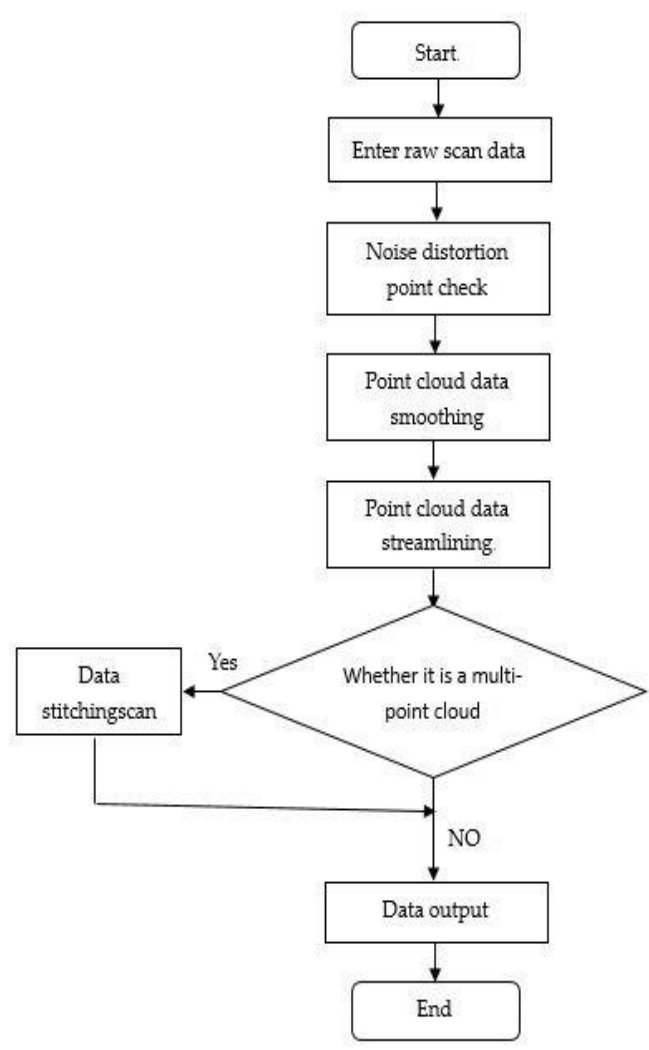

Figure 5. LiDAR data processing flow

2.3.3 Deformation information extraction method in LiDAR data: From the current development status of deformation monitoring using 3D laser scanning technology, mainly using three methods (Zhu et al., 2014):

\section{1) TARGET MARKING METHOD}

Multiple stable and evenly distributed targets are placed in the study area, and the study area is scanned at different time periods by a three-dimensional laser scanner, and the target center coordinates are presented in subsequent data processing, by comparing targets in multiple time periods. Changes in the central coordinates to extract change information. This method is similar to the traditional method, and although it has high precision, it cannot exert the advantages of 3D laser scanning.

\section{2) DEM DIFFERENCE METHOD}

The method is generally applied to the deformation monitoring of the surface, and the three-dimensional laser scanner is used to scan the research area in multiple time periods, and the multiphase point cloud data is pre-processed to generate a highprecision digital elevation model (DEM), and the time period is unified. In the DEM coordinate system, the DEM comparisons of different time periods are subtracted to obtain the corresponding settlement values of the entire region. Finally, using MATLAB and other software to make the deformation value into a section, a graph et al. to visually reflect the deformation.

\section{3) MODEL DIFFERENCE METHOD}


This method is used for deformation monitoring of space objects such as buildings and tunnels most of the time. The 3D laser scanner is used to acquire the point cloud data of the object. After pre-processing, the surface model of the object is generated. After the multi-period model is unified into a coordinate system, the models of different time periods are compared to obtain the deformation amount.

\section{RESULT ANALYSIS}

\subsection{InSAR deformation analysis}

The radar image of the study area was processed by the Permanent Scattering Synthetic Aperture Radar Interferometry (PS-InSAR) technique, and 20688 permanent scatterers (PS points) in the study area were obtained, from which 22 PS points of the Qianlong Garden Fuwangge Rockery were selected. In combination with the point cloud data collected by the three-dimensional laser scanner, 8 PS points are finally selected as data points for deformation analysis. As shown in Figure 6.
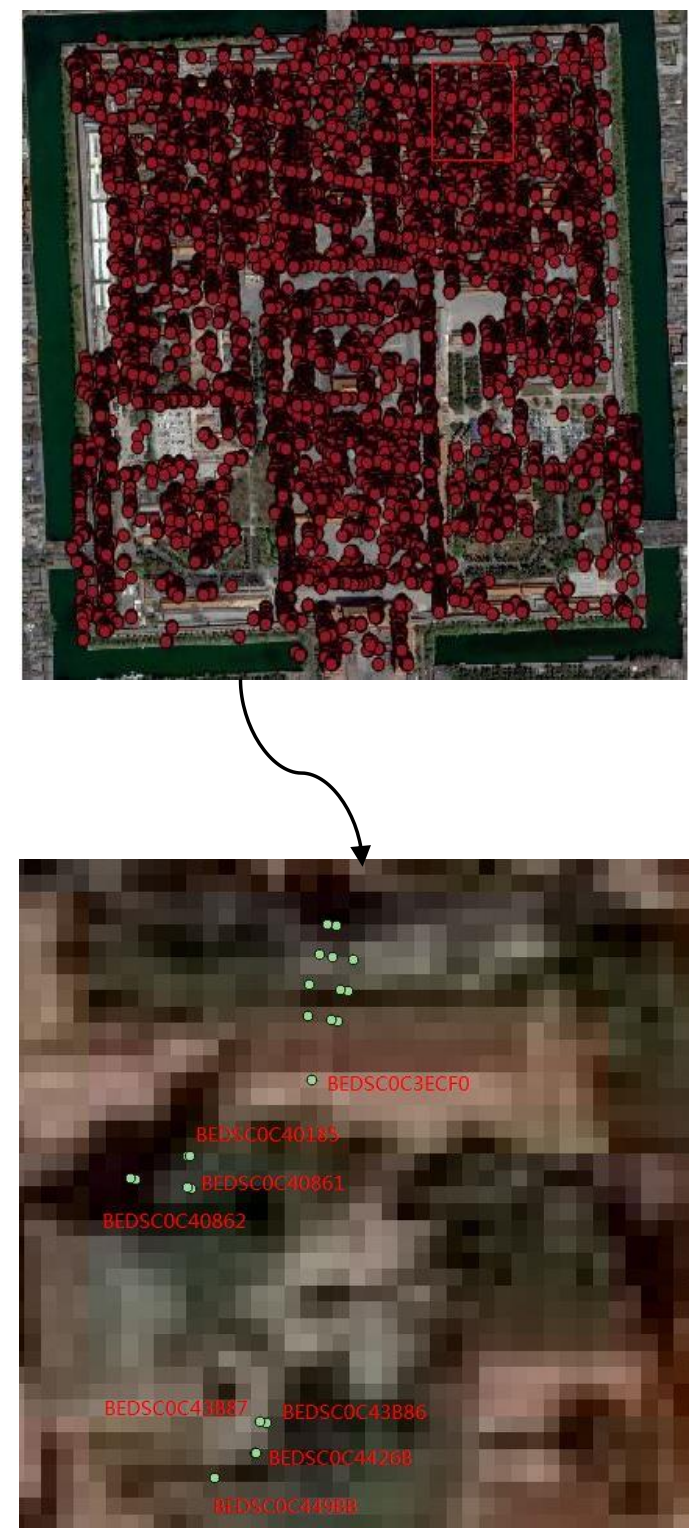

Figure 6. Distribution of PS points in the study area and Fuwangge Rockery
In order to facilitate the subsequent analysis work, the selected PS points are sequentially counter clockwise, as shown in Table 2.

\begin{tabular}{lll}
\hline 3ECF0 & 40862 & $4426 \mathrm{~B}$ \\
40185 & 43887 & $43 \mathrm{~B} 86$ \\
40861 & $449 \mathrm{BB}$ &
\end{tabular}

Table 2. Fuwangge Rockery Monitoring PS Point

As mentioned above, SAR radar images processed by PSInSAR technology generate a large number of PS points, which contain deformation information. A PS point in this article contains deformation information for 2011-2017. Based on this, in the QGIS, the deformation information contained in the PS points selected in Table 2 is directly generated into a line graph of the PS point deformation information, as shown in Figure 7.

From the deformation map of the eight PS points described above, since the time for starting the detection is 2011, the shape variable at the beginning of 2011 on the graph is 0 . The shape variables of the prosthetic mountains outside the Forbidden City in the study area are slowly increasing year by year. The maximum shape variable is about $1.5 \mathrm{~cm}$ in the comprehensive eight graphs. Taking Figure 7(a) as an example, BEDSC0C3ECF0 reached the largest deformation variable of about $1.4 \mathrm{~cm}$ in 2016 . The deformation of rockery shows a trend of increasing year by year. In order to visually observe the change law of the deformation information, a straight line is fitted when the curve is made by using the deformation information, and it can be seen that the deformation value fluctuates around the fitted straight line. Although the shape variables are different every month, it is foreseeable that the range of deformation varies substantially within the range of 0 $10 \mathrm{~mm}$, that is, the monthly deformation variable is within 10 $\mathrm{mm}$. This shows that the deformation of the rockery is a deformation under natural conditions rather than a severe deformation under extreme conditions. By analysing the deformation line diagrams of the above PS points, the following conclusions are drawn:

1) The main factor in the deformation of the rockery is the deformation of natural factors without the dramatic deformation under extreme conditions;

2) The maximum deformation variable of the rockery deformation is $15 \mathrm{~mm}$. It shows from the side that the deformation factor of the rockery belongs to the deformation under natural conditions, which provides important data support for the protection of the rockery;

3) The straight line fitted by the line graph can be seen that the shape variable of the rockery is increasing year by year. This is not uncontrollable, although the shape variable shows an upward trend as a whole, but since the shape variable is in the order of millimeters and the deformation is slow, this Providing sufficient time for the protection of the rockery;

4) The deformation monitoring of the rockery can also indirectly reflect the degree of deformation of the building around the rockery, providing an indirect method for obtaining deformation degree for deformation monitoring. 


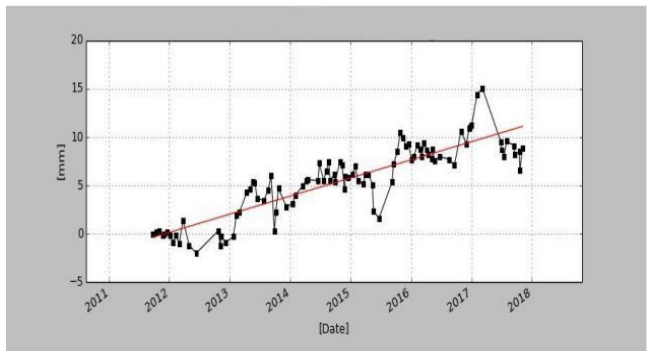

(a)

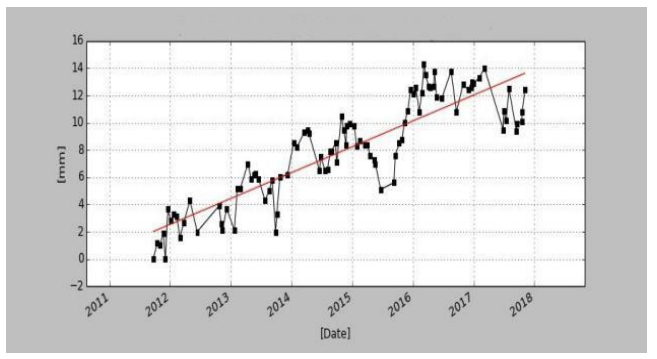

(c)

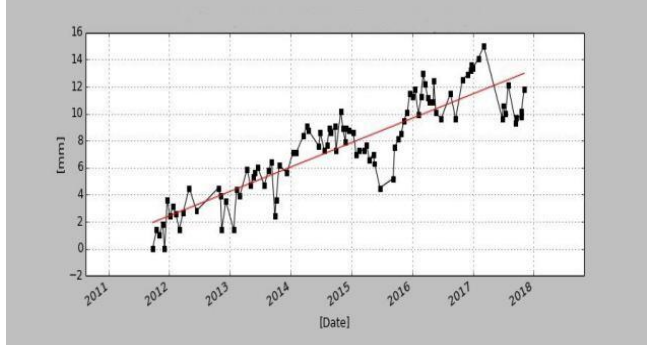

(e)

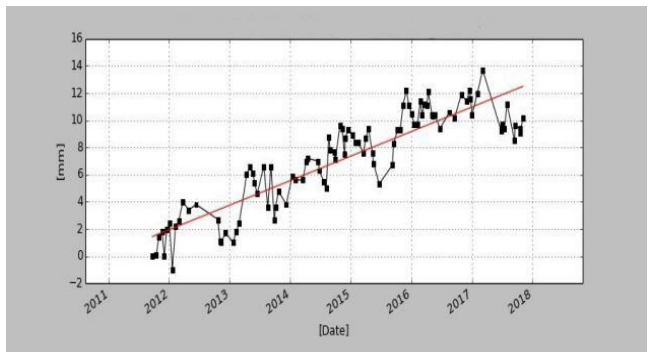

(g)

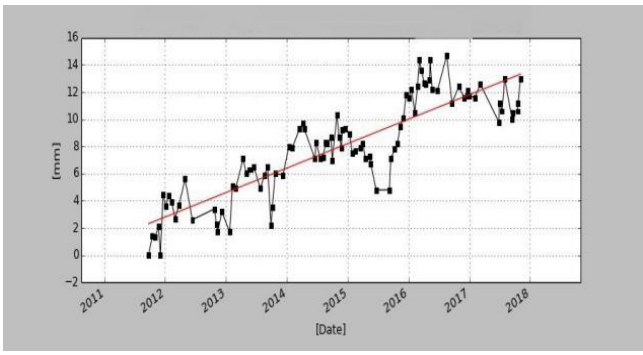

(b)

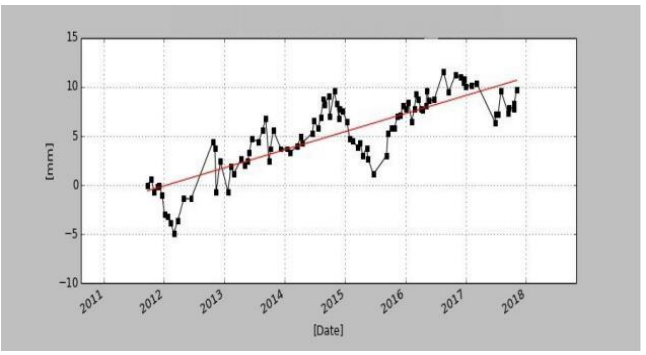

(d)

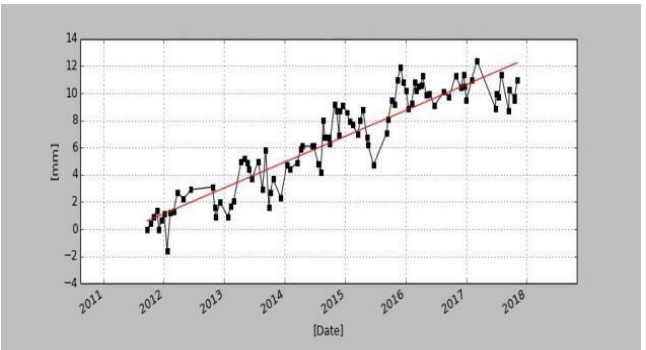

(f)

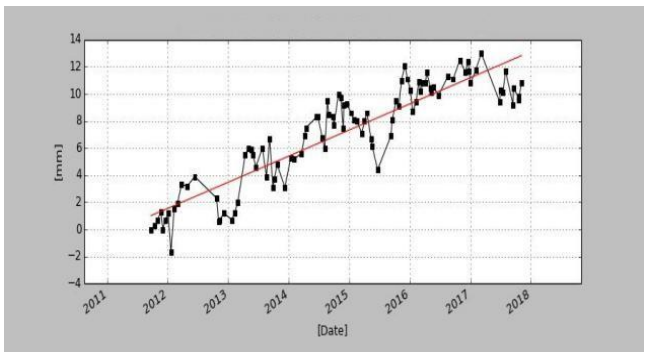

(h)

Figure 7. Study area selection PS point deformation information line diagram (a)3ECF0 deformation line diagram; (b)43B86 deformation line diagram; (c)43B87 deformation line diagram; (d)449BB deformation line diagram; (e)4426B deformation line diagram; (f)40185 deformation line diagram; (g)40861 deformation line diagram; (h)40862 deformation line diagram

\subsection{LiDAR deformation Analysis}

For obtaining the deformation information of rockery point cloud data, this paper adopts the method of comparing point cloud data with 3D model. Because single-site point cloud can't get the full view of rockery, this paper will register multi-site point cloud through the Pavilion. After the registration, the point cloud data in 2010 is packaged into a 3D model in Geomagic software, then the point cloud data in 2018 and the 3D model packaged by point cloud data in 2010 are compared by using the 3D analysis function of Geomagic software. Figure 8 shows the deviation distribution after the model and point cloud are superimposed.
As shown in Figure 8, the deformation information of the rockery can be extracted from the deviation map of the model and the point cloud. it can be seen that the left part of the rockery is mainly red with a shape variable of $5-10 \mathrm{~mm}$, which indicates that the shape variable of this part of the rockery is positive and the rockery has risen by $5-10 \mathrm{~mm}$; On the other hand, the right part of the rockery is mainly blue, and the deformation is also between $5-10 \mathrm{~mm}$, which indicates that the deformation of this part of the rockery is negative, and the rockery sinks by $5-10 \mathrm{~mm}$. In order to more intuitively see the segmentation of the various parts of the rockery and the shape variables, we give the top view after the superposition and the histogram of the deviation between the model and the rockery, as shown in Figure 9. 


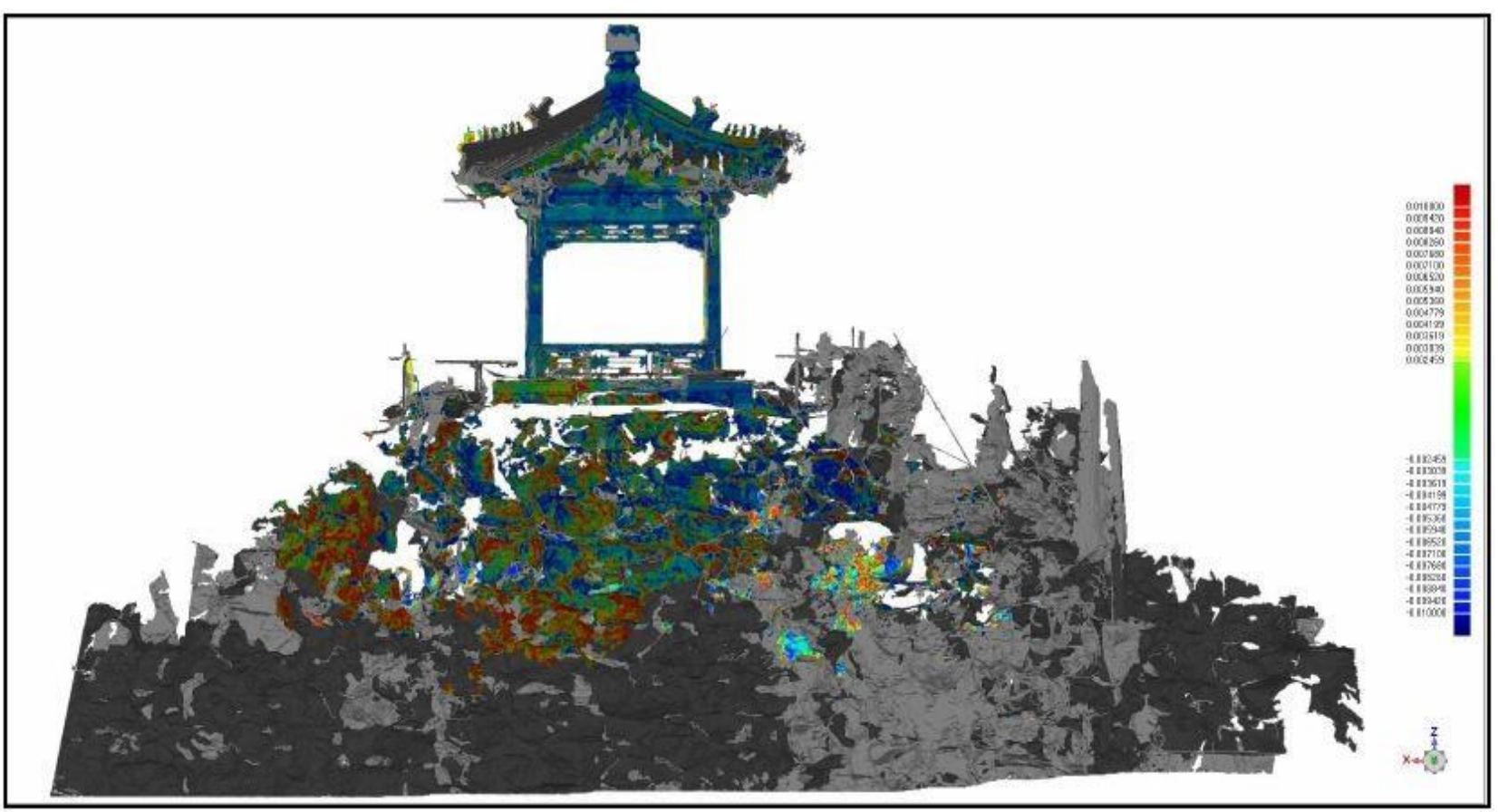

Figure 8. Model and point cloud overlay deviation distribution map

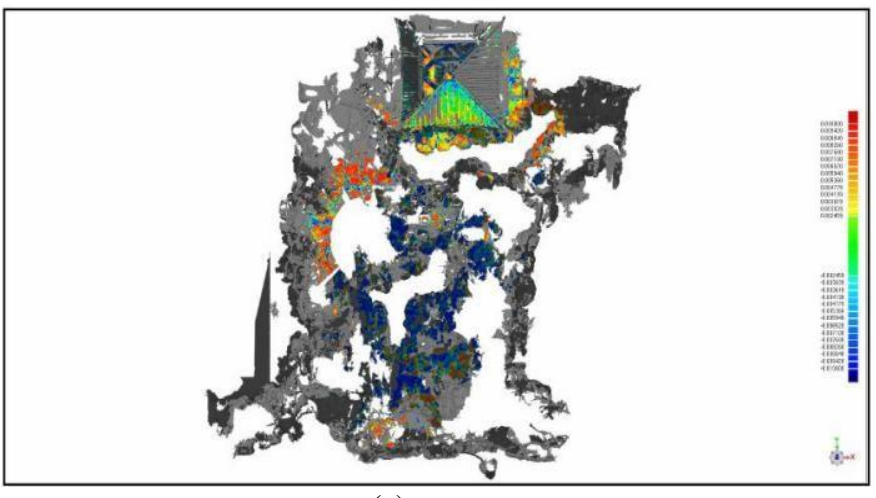

(a)

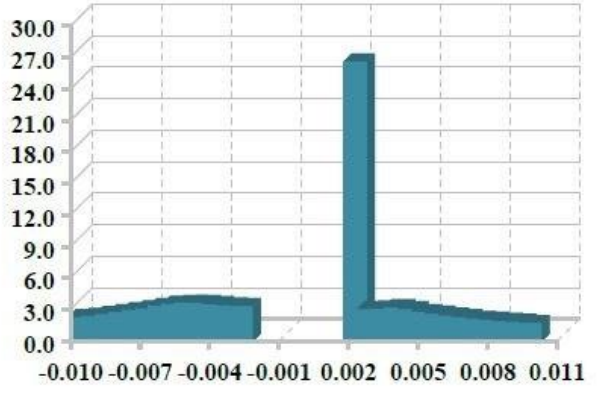

(b)

Figure 9. Top view and histogram after superposition (a) Top view of the model and point cloud overlay (b) Model and point cloud overlay histogram

From the superimposed top view and the deviation distribution histogram, it can be seen intuitively that in the rises left of the rockery, the shape variable is positive, mainly distributed in 2$11 \mathrm{~mm}$, indicating that the rockery in this part has a slight rise; in the right part of the rockery, the shape variable is negative, mainly distributed in $1-4 \mathrm{~mm}$, indicating that the rockery in this part has a slight sink.

From the above analysis, we can get the overall shape variables of different parts of the rocker, but we are not sure about the subtle changes in each part of the rockery. To solve this problem, we performed a $2 \mathrm{D}$ analysis. The rockery is divided into two sections from the vertical at $3 \mathrm{~m}$ intervals, so that more detailed deformation results can be obtained. The sectional view and its histogram are shown in Figure 10.

Figure 10(a) shows the part on the left side of the rockery in the above-mentioned 3D analysis which mainly shows an rises trend. As we can see from the section histogram, even if the whole part shows an upward trend, there are still a few parts with downward deformation. If only relying on $3 \mathrm{D}$ analysis, the subsidence deformation information of a few parts of the rockery on the left side will be ignored.

Figure 10(b) correspond to the part of the rockery that mainly sinks to the right side. It can be seen from the section histogram that the rockery in this part also contains a little rises deformation information under the overall sinks trend.

In summary, through the 2D and 3D analysis of the rockery, we not only get the deformation information of the rockery, but also analyze the deformation of each part of the rockery in a more detailed way. The deformation of the rockery is not a single slight rise or sink. The deformation of the rockery is comprehensive. Deformation exists in all parts of the rockery. 

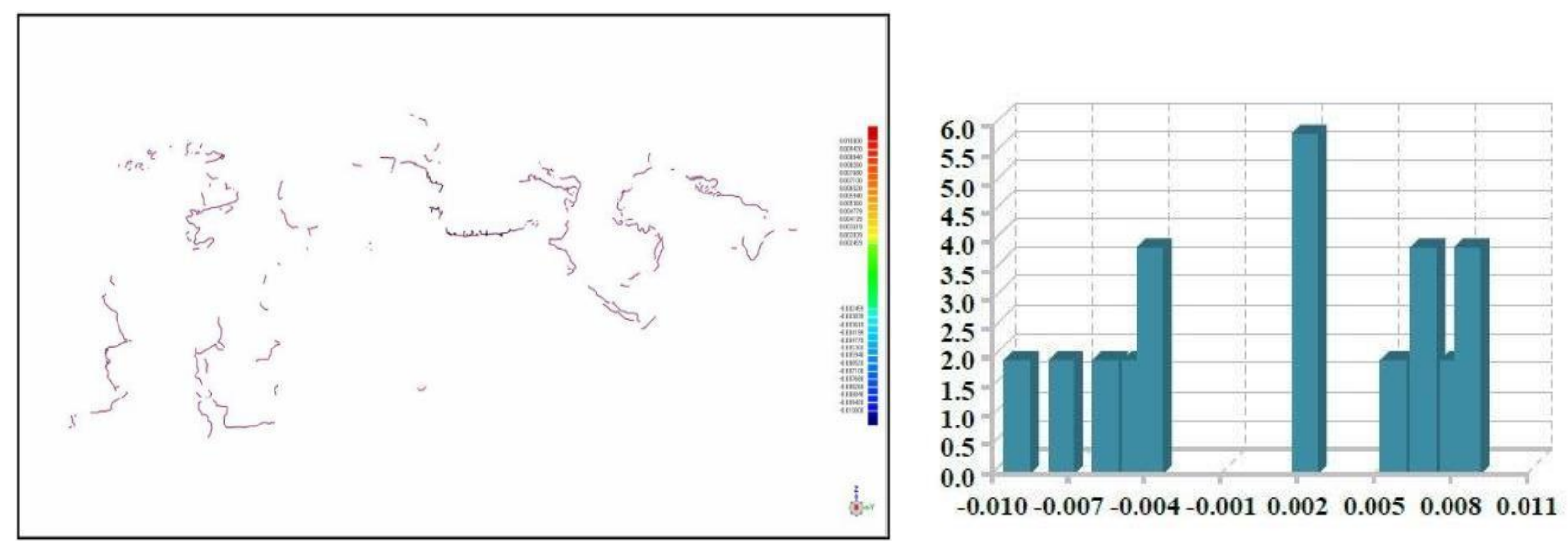

(a)
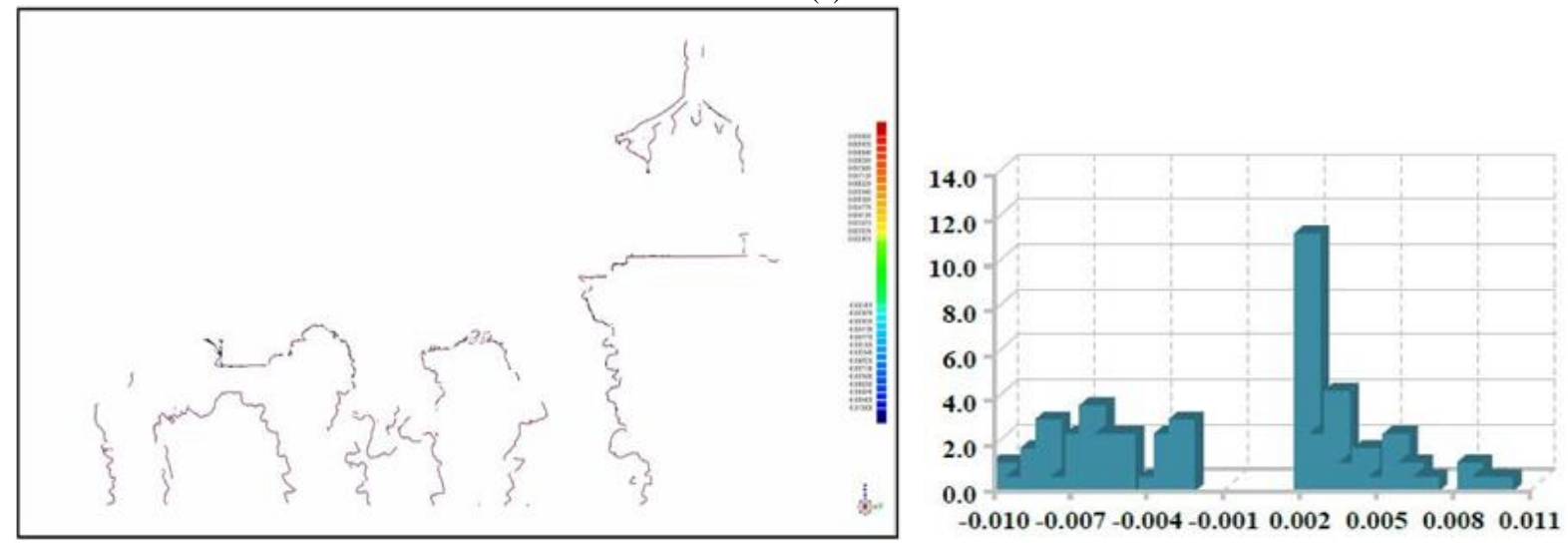

(b)

Figure 10. $X=-3$ and $X=3$ make the rockery profile and histogram; (a)Sectional view and histogram of $X=-3(b)$ Sectional view and histogram of $\mathrm{X}=3$

\subsection{Comprehensive analysis of InSAR deformation and LiDAR deformation results}

Sections 3.1 and 3.2 respectively analyze the deformation of the rockery at Fuwangge in Qianlong Garden of the Forbidden City from two data sources. The deformation of the rockery obtained by InSAR technology is only a single point and an overall deformation. The deformation analysis based on this can not obtain the specific deformation of each part of the rockery. On the contrary, LiDAR technology has higher resolution, so we can study the specific deformation of each part of rockery, but its point accuracy is lower than InSAR technology. Therefore, we can get more convincing results by evaluating the deformation results obtained by the two methods. The maximum shape variable obtained by InSAR technology is $13 \mathrm{~mm}$, and the maximum shape variable obtained by LiDAR is $11 \mathrm{~mm}$, which is consistent with our expected results. At the same time, the line chart in Figure 7 also reflects that the deformation of rockery is not simply rising or sinking, which is consistent with the deformation of rockery obtained by LiDAR technology.

\section{CONCLUSIONS}

This article has done these works:

(1) InSAR and LiDAR techniques were used to monitor the deformation of the rockery in Qianlong Garden respectively

(2) The deformation change of rockery from 2010 to 2018 was analyzed by combining the two technologies

(3) It provides a new method for deformation monitoring of architectural heritage
This article also has some deficiencies:

(1) Only the vertical deformation of the rockery in Qianlong Garden is evaluated

(2) LiDAR point cloud registration accuracy needs to be improved

Through the above experiments and analysis of experimental results, it can be concluded that the method of using LiDAR technology combined with InSAR technology for deformation monitoring is feasible. At the same time, we also found that LiDAR technology used for deformation monitoring can better reflect the subtle deformation of rockery, which is not achieved by InSAR technology. Therefore, the combination of InSAR technology and LiDAR technology for deformation monitoring provides a new way for related work in the future.

\section{ACKNOWLEDGMENTS}

This work was supported by National Natural Science Foundation of China (No. 41971350), The Beijing Advanced Innovation Center for Future Urban Design Project (UDC2019031724), the Development Project of Beijing Municipal Science and Technology (No. KM201510016016, No. KM201810016013), The Fundamental Research Funds for Beijing Universities (No. X18050, No. X18001, No. X18299), Special Entrustment Project of Scientific Research Fund of Beijing University of Civil Engineering and Architecture (No. KYJJ2017024) and Beijing Institutions of higher learning innovation ability enhancement (No. TJSH20161001602). 


\section{REFERENCES}

Guo Zonghe. Practical Survey of Engineering Measurement [M]. China Electric Power Press,2013.

Zhu Shengtao. Research on the Application of Ground 3D Laser Scanning Technology in the Monitoring of Earth Surface Deformation [D]. Xi'an: Chang'an University,2013.

Zhang Lishuo, Cheng Xiaojun. Tunnel deformation analysis method based on laser point cloud [J]. Chinese Journal of Laser, 2018,45 (04): 225-230.

Yang Yonglin, the first application of laser scanning to protect large-scale cultural relic sites [N]. People's Daily, 2006, 4(5).

Chang Ming, Pan Lijun, Meng Xiangang, Xu Yujian, Xu Kai. Research on slope deformation monitoring based on 3D laser scanner [J]. Journal of Geodesy and Geodynamics, 2019, 39 (05): 533-537.

Wang Yanmin, Kui Hui, Wang Guoli, Hu Chunmei. Application of ground lidar technology to deformation analysis of Baiju Temple auspicious multi-door tower [J]. Journal of Beijing Institute of Civil Engineering and Architecture, 2011, 27 (04): $11-15+45$.

Li Yan, Lu Xiaoping, Zhu Ningning, Lu Yao, Wu Yongbin, Li Guoqing. Laser Point Cloud Continuous Extraction and Deformation Analysis of Tunnel Sections [J]. Acta Geodaetica et Cartographica Sinica, 2015, 44 (09): 1056-1062.

Guo Ming, Shao Tianyi, Gao Chutian, Zhao Jianghong. High precision deformation analysis of rock material for simulating corrosion[J]. Science of Surveying and Mapping, 2020, 45(02): 85-91.

Wang Guoli, Wu Guikai, Wang Yanmin, Guo Ming, Zhao Jianghong, Gao Chao. Deformation Monitoring of Ancient Pagoda with Multi-source Data[J]. Journal of Geo-information Science, 2018, 20(04): 496-504.

Gao Chao, Wang Guoli, Wang Yanmin, Zhao Jianghong. The status and development of digital phenotypic monitoring technology for architectural heritage $[\mathrm{J}]$. Science of Surveying and Mapping, 2019, 44(05): 85-92.

Pagounis Vassilios, Tsakiri Maria, Palaskas Spiridon et al. 3D Laser Scanning for Road Safety and Traffic Accident Reconstruction [C]. Shaping the Change XIII FIG Congress Munich.Germany, 2006: 8-13.

Qin Liming. The Analysis of SAR Technology in Deformation Monitoring[J]. Beijing Surveying and Mapping, 2015, (5): 6669.
Zhu Jianjun, Li Zhiwei, Hu Jun. Research Progress and Methods of InSAR for Defomation Monitoring [J]. Acta Geodaetica et Cartographica Sinica, 2017(10): 519-535.

Zhang Bei. Research on surface deformation monitoring of cities and mining areas based on PS-DInSAR technology [D]. Taiyuan University of Technology, 2019.

Huang Jilei. Research on key issues of Pixel-tracking of SAR images for large gradient deformation monitoring in mining areas [D]. China University of Mining and Technology, 2017.

Ferretti A, Prati C, Rocca F. Nonlinear Subsidence Rate Estimation Using Permanent Scatterers in Differential SAR Interferometry $[\mathrm{J}]$. IEEE Transaction on Geoscience and Remote Sensing, 2000, 38(5): 2202 -2212.

Ferretti A, Prati C, Rocca F. Permanent Scatterers in SAR Interferometry $[\mathrm{J}]$. IEEE Transactions on Geoscience and Remote Sensing, 2001, 39(1): 8-20.

Chen Qiang. Detection Regional Ground Deformation by Differential SAR Interferometry Based on Permanent Scatterers [D]. Chengdu: Southwest Jiaotong University,2006.

Xiong Wenxiu, Feng Guangcai, Li Zhi wei, et al. High Quality Targets Selection in SBAS-InSAR Technique by Considering Temporal and Spatial Characteristic[J]. Acta-Geodaetica et al. Cartographica Sinica, 2015, 44(11): 1246-1254.

Jiang Yanan. Research on SAR deformation observation, interpretation and data assimilation in geological disaster monitoring [J]. Acta Geodaetica et Cartographica Sinica, 2018, 47 (10): 1425.

Diao Xinpeng. Research on SAR/InSAR monitoring technology of large-scale surface deformation in mining area [D]. China University of Mining and Technology, 2018.

Kampes B M, Hanssen R F. Ambiguity Resolution for Permanent Scatterer Interferometry[J]. IEEE Transactions on Geoscience and Remote Sensing, 2004, 42(11): 2446-2453.

Hopper A, Zebker H A. Phase Unwrapping in Three Oimensions with Application to INSAR Time Series [J]. Journal of the Optical Society, 2007, 24(9): 2737-2747.

Li Deren, Liao Mingsheng, Wang Yan. Progress of Permanent Scatterer Interferometry $[\mathrm{J}]$. Geomatics and Information Science of Wuhan University, 2004, 29(8): 664-668.

Zhu Lei, Wang Jian, Bi Jingxue. Application of 3D Laser Scanning Technology in Deformation Monitoring [J]. Beijing Surveying and Mapping,2014:82-86. 